\title{
A FIXED-POINT THEOREM FOR DEFINABLY AMENABLE GROUPS
}

\author{
JUAN FELIPE CARMONA, KEVIN DÁVILA, ALF ONSHUUS, \\ AND RAFAEL ZAMORA
}

\begin{abstract}
We prove an analogue of the fixed-point theorem for the case of definably amenable groups.
\end{abstract}

\section{INTRODUCTION}

In the context of locally compact groups, there are several properties that have been proved to be equivalent to amenability 1 . One of those is the fixedpoint property (a generalization of the Markov-Kakutani theorem), which states that any affine continuous action over a compact convex subset of a locally convex vector space has a fixed point.

The analogue of amenability for definable groups was defined by [4] as follows: A definable group is definably amenable if there is a left-invariant finitely-additive probability measure on all its definable subsets (a left invariant global Keisler Measure). It is known that every stable group is definably amenable [5], and that groups definable in a dependent theory are definably amenable if and only if they have an $f$-generic type [4] (called strongly $f$-generic types in [1]).

In this note we study definably amenable groups and prove that definable amenability is equivalent to a fixed-point condition. We do not assume that the group is in any "good" class in the sense of Classification Theory.

The paper is divided as follows: In Section 2, we study the $\sigma$-topology of a definable group, which allows us to study the $\sigma$-algebra generated by definable sets (and measures on it) using topological tools. The notion of $\sigma$-topology allows us to see definable sets and topological spaces as objects of the same category. In particular, we prove that the $\sigma$-continuous image of a definable subset of an $\omega_{1}$-saturated structure in a Polish space is compact.

In 2.3 we show that the existence of an invariant mean is equivalent to the existence of an invariant measure, when the group is $\omega_{1}$-saturated. This would be an immediate corollary of Hahn-Kolmogorov Theorem.

\footnotetext{
${ }^{1}$ In this paper we say that a topological group is amenable if it admits a left invariant finitely additive probability measure on the Borel subsets.
} 
In Section 3 we prove the main result of the paper:

Theorem. The following are equivalent:

(1) $G$ is an $\omega_{1}$-saturated definably amenable group.

(2) If $\pi$ is a linear action of $G$ into a convex compact subset $Y$ of a locally convex topological vector space $V$ such that

(a) $\pi_{g}$ is continuous for every $g \in G$.

(b) $\pi_{k}$ is $\sigma$-continuous for some $k \in Y$.

Then $\pi$ has a fixed point.

When working in with the definable topology $\sigma$-continuity is equivalent to logic-continuity (see Theorem 2.14), a concept which may be more familiar for model theorists. We state everything in terms of $\sigma$-continuity because it allows for cleaner proofs.

\section{The $\sigma$-TOPOLOGY OF A FIRST-ORDER STRUCTURE}

In this section we introduce the notion of $\sigma$-space, which will allow us to consider definable sets in first-order structures, open sets in topological spaces, and even Borel measurable sets in a $\sigma$-algebra, as objects of the same category.

\subsection{The category of $\sigma$-spaces.}

Definition 2.1. Let $X$ be a non-empty set, we say that $\tau \subset \mathcal{P}(X)$ is a $\sigma$-topology on $X$ if:

(1) $\emptyset, X$ are in $\tau$.

(2) $\tau$ is closed under finite intersections and countable unions.

We say that $(X, \tau)$ is a $\sigma$-space if $\tau$ is a $\sigma$-topology on $X$.

The definitions of $\sigma$-open, $\sigma$-closed and $\sigma$-continuity are the natural ones.

\section{Example 2.2.}

- Any topological space is a $\sigma$-space.

- Any $\sigma$-algebra is a $\sigma$-space (although here there is no distinction between $\sigma$-open and $\sigma$-closed sets).

Definition 2.3. A collection $B$ of subsets of $X$ is a basis for a $\sigma$-topology $\tau$ if every $U \in \tau$ is a countable union of sets in $B$.

Remark 2.4. The family $B$ is a basis for some $\sigma$-topology if and only if it is closed under finite intersections.

Let $M$ be any structure. If $X$ is an $A$-definable set in a structure $M$, then $\operatorname{Def}_{A}(X)$ satisfies the conditions of the previous remark and therefore is a basis for a $\sigma$-topology on $X$.

From now on, we will consider any structure $M$ as a $\sigma$-space, where the $\sigma$-topology is generated by $\operatorname{Def}_{M}(M)$. 
Example 2.5. Let $\mathbb{R}^{*}$ be the non-standard reals (which is an $\omega_{1}$ saturated extension of $\mathbb{R})$ and let st $: \mathbb{R}^{*} \rightarrow \mathbb{R} \cap\{-\infty, \infty\}$ the standard map. Then st is $\sigma$-continuous (seeing $\mathbb{R}^{*}$ as a first-order structure and $\mathbb{R} \cap\{-\infty, \infty\}$ as the usual topological space). Notice that the image of $\mathbb{R}^{*}$ is compact.

Remark 2.6. Any structure $M$ can be seen as a subset of its (Stone space) space of types $S(M)$ (the Stone space of $\operatorname{Def}_{M}(M)$ ), where the inclusion $i$ is given by $i(m)=t p(m / M)$. The topology of $S(M)$ is compact but is discrete when restricted to $M$, therefore the inclusion is not $\sigma$-continuous in general.

We are interested in treating first-order structures as $\sigma$-spaces. The next definition will characterize saturation in terms of the $\sigma$-topology (this is merely a translation, but it would be very useful in order to characterize the image of saturated structures under $\sigma$-continuous functions).

Definition 2.7. We say that a $\sigma$-topology $(X, \tau)$ is countably compact if every countable open cover of $X$ has a finite subcover.

By definition of saturation, we have the following:

Remark 2.8. A structure $M$ is $\omega_{1}$-saturated if and only if it is countably compact as a $\sigma$-space.

The image of a countably compact space under a $\sigma$-continuous function is countably compact. Therefore we have the following result.

Theorem 2.9. If $X$ is countably compact, $Y$ is a second-countable topological space and $f: X \rightarrow Y$ is $\sigma$-continuous, then $f(X)$ is compact in $Y$.

2.2. Definable Functions. We remark that the notion of $\sigma$-continuity has some similarities with the notion of definable function given in [3]:

Definition 2.10 (Gismatullin, Penazzi, Pillay). Let $M$ be any structure, let $X$ be an $M$-definable set in a saturated extension $M^{*}$ and $C$ a compact space. A function $f: X \rightarrow C$ is definable if for any closed set $D$, its preimage is type-definable over $M$.

It may be worth to clarify how they differ from each other.

Notice that the pre-image of closed sets by definable functions are sets that are type-definable over some small model $M$, while the pre-image of closed sets by $\sigma$-continuous functions are type-definable over the whole model, but only with countably many formulas. It is clear then that $t p: X \rightarrow S_{M}(X)$ is a definable function but it is not $\sigma$-continuous in general.

Our definition comes from the fact that we need to deal with the $\sigma$-algebra generated by all the definable sets of a group, so we cannot relativize to a small model. On the other hand, the topology generated by the definable sets is the discrete one and the notion of definable function, without relativizing to a small model, is no longer useful. Therefore, we need to take a weak notion of topology (i.e $\sigma$-topologies) and to study $\sigma$-continuity instead. 
2.3. Measures. The $\sigma$-algebra generated by the definable sets of a structure $M$ is precisely the Borel subsets of $M$ when is seen as a $\sigma$-space.

Definition 2.11. Let $M$ be any structure, a Keisler measure on $M$ is a finitely-additive probability measure $\mu_{0}$ over $\operatorname{Def}(M)$.

We recall here the Hahn-Kolmogorov Theorem, which will allow us to extend $\mu_{0}$ to a $\sigma$-additive measure over the $\sigma$-algebra generated by $\operatorname{Def}(M)$.

Theorem 2.12 (Hahn-Kolmogorov). If X has a finitely-additive probability measure $\mu_{0}$ over an algebra of subsets $B$, such that:

For every countable family $\left\{D_{i}\right\}_{i<\omega}$ of disjoint subsets in $B$ such that $\bigcup_{i<\omega} D_{i} \in B$, we have that $\mu_{0}\left(\bigcup_{i<\omega} D_{i}\right)=\sum_{i<\omega} \mu_{0}\left(D_{i}\right)$.

Then, there is a unique measure $\mu$ over the $\sigma$-algebra generated by $B$ that extends $\mu_{0}$.

Theorem 2.13. Every Keisler measure $\mu_{0}$ over an $\omega_{1}$-saturated structure $M$ can be extended to a $\sigma$-additive measure over the $\sigma$-algebra generated by $\operatorname{Def}(M)$.

Proof. We need to check that $\left(M, \operatorname{Def}(M), \mu_{0}\right)$ satisfies the hypothesis of the Hahn-Kolmogorov Theorem: Let $\left\{D_{i}\right\}_{i<\omega}$ be a family of disjoint subsets in $\operatorname{Def}(M)$ such that $\bigcup_{i<\omega} D_{i}=D \in \operatorname{Def}(M)$. By $\omega_{1}$-saturation only a finite number of $D_{i}$ 's are non empty, hence the conclusion follows.

Let us note that if $M$ is $\omega_{1}$-saturated and has a Keisler measure, then it may be seen as a measurable space, where the $\sigma$-algebra is generated by the definable sets. Moreover, if $X$ is a topological space with a Borel-measure, then every $\sigma$-continuous function $f: M \rightarrow X$ is measurable in the usual sense.

2.4. Polish spaces and $\sigma$-continuity. We establish several characterizations of $\sigma$-continuity over Polish spaces. Recall that a Polish space may be characterized as a second-countable locally-compact Hausdorff space.

Theorem 2.14. Let $f: M \rightarrow X$ be a function from an $\omega_{1}$-saturated structure $M$ to a Polish-space $X$. The following are equivalent:

- $f$ is $\sigma$-continuous.

- For every $K \subset U$ in $X$, with $K$ compact and $U$ open, there exists a definable set $D$ such that

$$
f^{-1}(K) \subset D \subset f^{-1}(U) .
$$

- For every $x \in X$ and for every open $U$ containing $x$, there exists $K \subset U$ a compact neighborhood of $x$ and $D$ definable such that

$$
f^{-1}(K) \subset D \subset f^{-1}(U) .
$$


Proof. (1) $\Rightarrow(2)$ : By $\sigma$-continuity, $f^{-1}(K)$ is $\sigma$-closed and $f^{-1}(U)$ is $\sigma$-open. Therefore, by compactness and $\omega_{1}$-saturation there must be a definable set in between.

$(2) \Rightarrow(3)$ : This is due to the local-compactness of $X$.

$(3) \Rightarrow(1)$ : Let $U$ be an open set. For every $x \in U$, we can find a neighborhood $K_{i} \subset U$ and $D_{x}$ definable such that $f^{-1}\left(K_{x}\right) \subset D_{x} \subset f^{-1}(U)$. Notice that $\left\{K_{x}\right\}$ is a cover of $U$ so we may find a countable subcover $\left\{K_{i}\right\}_{i<\omega}$ of $U$ (this cover exists because $X$ is Polish). By hypothesis, for every $i$ there exists $D_{i}$ definable such that $f^{-1}\left(K_{i}\right) \subset D_{i} \subset f^{-1}(U)$.

Clearly $f^{-1}(U)=\bigcup_{i<\omega} D_{i}$.

Lemma 2.15. If $f_{1}, f_{2}: M \rightarrow \mathbb{R}$ are $\sigma$-continuous, then both $\left(f_{1}, f_{2}\right): \mathcal{G} \rightarrow$ $\mathbb{R}^{2}$ and $f_{1}+f_{2}$ are $\sigma$-continuous.

Proof. Let $U$ be an open set of $\mathbb{R}^{2}$ and let $\bar{x}=\left(x_{1}, x_{2}\right) \in U$. Take any box $U_{1} \times U_{2}$ of open sets such that $\bar{x} \in U_{1} \times U_{2} \subset U$ and take $K_{1} \times K_{2}$ a box of compact sets where $K_{i} \subset U_{i}$ is a neighbourhood of $x_{i}$. By $\sigma$-continuity of $f_{1}$ and $f_{2}$ we can find $D_{1}$ and $D_{2}$ such that $f_{1}^{-1}\left(K_{i}\right) \subset D_{i} \subset f_{2}^{-1}\left(U_{i}\right)$. Therefore,

$$
\left(f_{1}, f_{2}\right)^{-1}\left(K_{1} \times K_{2}\right) \subset D_{1} \cap D_{2} \subset\left(f_{1}, f_{2}\right)^{-1}\left(U_{1} \times U_{2}\right)
$$

and therefore $\left(f_{1}, f_{2}\right): \mathcal{G} \rightarrow \mathbb{R}^{2}$ is $\sigma$-continuous.

Now, notice that

$$
f_{1}+f_{2}: M \stackrel{\left(f_{1}, f_{2}\right)}{\longrightarrow} \mathbb{R}^{2} \stackrel{+}{\longrightarrow} \mathbb{R} .
$$

Since the composition of $\sigma$-continuous functions is $\sigma$-continuous, we have that $f_{1}+f_{2}$ is $\sigma$-continuous.

Remark 2.16. If $D$ is a definable set, then its characteristic function

$$
\mathbf{1}_{D}: M \rightarrow \mathbb{R}
$$

is $\sigma$-continuous.

Theorem 2.17. Let $M$ be a saturated structure. Then the space $C_{\sigma}(M)$ of $\sigma$-continuous functions of $M$ over $\mathbb{R}$ equipped with the supremum norm is a Banach space.

Proof. By Lemma 2.15 we know that the $\sigma$-continuous functions form a vector space. We just need to show that Cauchy sequences converge.

Let $\left\langle f_{i}\right\rangle_{i \in \omega}$ be a sequence of $\sigma$-continuous functions from $M$ to $\mathbb{R}$ such that for every $\epsilon$ there is an $N$ such that for all $x \in M$, for all $i, j>N$ we have $\left|f_{i}(x)-f_{j}(x)\right|<\epsilon$. Since $\mathbb{R}$ is complete the function $F(x)=\lim _{i \rightarrow \omega} f_{i}(x)$ exists.

We need to show that $F$ is $\sigma$-continuous. Let $U$ be an open set in $\mathbb{R}$ and take $V:=B_{\epsilon / 8}(a)$ such that $B_{\epsilon}(a) \subset U$. By Theorem 2.14, it is enough to show that there is a definable $D$ such that $f^{-1}(\bar{V}) \subseteq D \subseteq F^{-1}(U)$. 
Let $N$ be such that for $i, j \geq N$ we have $\left|f_{i}(x)-f_{j}(x)\right|<\epsilon / 8$ which implies that $\left|f_{N}(x)-F(x)\right| \leq \epsilon / 8$. By $\sigma$-continuity of $f_{N}$, let $D$ be a definable set such that

$$
f_{N}^{-1}\left(\overline{B_{\epsilon / 4}(a)}\right) \subseteq D \subseteq f_{N}^{-1}\left(B_{\epsilon / 2}(a)\right)
$$

Now, if $x \in D$ we have $f_{N}(x) \in B_{\epsilon / 2}(a)$ and $\left|f_{N}(x)-F(x)\right| \leq \epsilon / 8$ which by triangle inequality implies $F(x) \in B_{5 \epsilon / 8}(a)$.

On the other hand, if $F(x) \in \overline{B_{\epsilon / 8}(a)}$, again by triangle inequality we have $f_{i}(x) \in B_{\epsilon / 4}(a)$, hence $\left.x \in D\right)$. It follows that

$$
F^{-1}(\bar{V}) \subseteq D \subseteq F^{-1}(U)
$$

as required.

\section{Definable amenability and the FiXed-Point PRoperty}

Definition 3.1. A definable group $\mathcal{G}$ is definably amenable if it has a leftinvariant Keisler measure.

If $\mathcal{G}$ is an $\omega_{1}$-saturated definably amenable group, by Theorem 2.13 we know that its Keisler measure $\mu_{0}$ can be extended to a measure $\mu$ on $\mathcal{B}$, the $\sigma$-algebra generated by $\operatorname{Def}(M)$. It is easy to see that $\mu$ is left-invariant.

Notice that $\mu$ can be seen as a linear functional from $\mathcal{S}(\mathcal{G})$ to $\mathbb{R}$, where $\mathcal{S}(\mathcal{G})$ is the set of simple measurable functions from $\mathcal{G}$ to $\mathbb{R}$ :

$$
\mathcal{S}(\mathcal{G}):=\left\{\sum_{i \leq n} a_{i} \mathbf{1}_{B_{i}} \mid B_{i} \in \mathbb{B}, a_{i} \in \mathbb{R}, n<\omega\right\}
$$

It is well known that $\mathcal{S}(\mathcal{G})$ is a dense subset of the Banach space of essentially bounded measurable functions $L^{\infty}(\mathcal{G})$.

With this in mind, we can prove now the following theorem:

Theorem 3.2. If $\mathcal{G}$ is an $\omega_{1}$-saturated definably amenable group, then there exists an invariant mean on $L^{\infty}(\mathcal{G})$.

Proof. Since $\mathcal{S}(\mathcal{G})$ is dense in $L^{\infty}(\mathcal{G})$, the left-invariant mean $\mu \in \mathcal{S}(\mathcal{G})$ has a unique extension to a mean $\mu^{\prime} \in L^{\infty}(\mathcal{G})$. Notice that $\mu^{\prime}\left(\mathbf{1}_{\mathcal{G}}\right)=1$. It only remains to show that $\mu^{\prime}$ is invariant:

Let $f \in L^{\infty}(\mathcal{G})$ and $g \in G$. By density, for every $\epsilon>0$ there exists $f^{\prime} \in \mathcal{S}(\mathcal{G})$ such that $\left|f-f^{\prime}\right|<\epsilon / 2$. Therefore

$$
\begin{gathered}
\left|\mu^{\prime}\left({ }_{g} f-f\right)\right| \leq\left|\mu^{\prime}\left(g\left(f-f^{\prime}\right)\right)\right|+\left|\mu^{\prime}\left({ }_{g} f^{\prime}-f^{\prime}\right)\right|+\left|\mu^{\prime}\left(f^{\prime}-f\right)\right| \\
\leq 2\left|f-f^{\prime}\right|<\epsilon .
\end{gathered}
$$

Definition 3.3. Let $\pi: \mathcal{G} \times X \rightarrow X$ be a group action. We say that $\pi$ is separately $\sigma$-continuous if

$$
\pi_{g}: X \rightarrow X ; \pi_{g}(x)=\pi(g, x)
$$


and

$$
\pi_{x}: G \rightarrow X ; \pi_{x}(g)=\pi(g, x)
$$

are $\sigma$-continuous for every $g \in \mathcal{G}$ and every $x \in X$. This implies in particular that $\pi_{g}$ is an homeomorphism for every $g$.

Definition 3.4. Let $V$ be a vector space, $F$ a family of subsets of $V$, and $U \subset V$. We say that $F$ is $U$-fine if every element of $F$ is contained in a translate of $U$.

Theorem 3.5 (Fixed-point theorem). The following are equivalent:

(1) $G$ is an $\omega_{1}$-saturated definably amenable group.

(2) If $\pi$ is a linear action of $G$ into a convex compact subset $Y$ of a locally convex topological vector space $V$ such that

(a) $\pi_{g}$ is continuous for every $g \in G$.

(b) $\pi_{k}$ is $\sigma$-continuous for some $k \in Y$.

Then $\pi$ has a fixed point.

Proof. $(1) \rightarrow(2)$ : This is very similar to the proof in [6]. Let $k \in Y$ such that $\pi_{k}$ is $\sigma$-continuous. Let

$F=\left\{U_{1}, \ldots, U_{n}\right\}$ be a minimal open cover of $Y$, define

$S_{1}=\pi_{k}^{-1}\left(U_{1}\right), \ldots, S_{i}=\pi_{k}^{-1}\left(U_{i} \backslash\left(U_{1} \cup \ldots \cup U_{i-1}\right)\right) \ldots S_{n}=\pi_{k}^{-1}\left(U_{n} \backslash\left(U_{1} \cup \ldots \cup U_{n-1}\right)\right)$

and $\mu_{U_{i}}=\mu\left(S_{i}\right)$ for every $i \leq n$.

The numbers $\mu_{U_{i}}$ depend on the order of $F$, however $\Sigma_{i} \mu_{U_{i}}=1$ for any order of $F$.

Let $D$ be the set of finite minimal open covers of $Y$ ordered by refinement.

For every $F \in D$, let $\left\{s_{U}^{F}\right\}_{U \in F}$ be a set of points such that $s_{U}^{F} \in U \cap Y$, and define the function.

$$
\begin{aligned}
\Phi: D & \longrightarrow Y \\
F & \mapsto \sum_{U \in F} \mu_{U} \cdot s_{U}^{F},
\end{aligned}
$$

The expression defining $\Phi(G)$ is a convex linear combination, hence an element of $Y$.

Claim 3.6. If $F_{1} \preceq F_{2}$ are covers in $D, V$ is a symmetric convex neighborhood of 0 and $F_{1}$ is $V / 2$-fine, then $\Phi\left(F_{1}\right)-\Phi\left(F_{2}\right) \in V$.

Proof. We only show the case when $F_{1}=\{U\}$, other cases will be similar:

Since $F_{1}$ is $V / 2$-fine, there is some element $t$ such that $t+V / 2 \supseteq U$. Let $\Phi\left(F_{1}\right)=s_{U}^{F_{1}}$ and $\Phi\left(F_{2}\right)=\sum_{U^{\prime} \in F_{2}} \alpha_{U^{\prime}} \cdot s_{U^{\prime}}^{F_{2}}$, then we have

$$
\Phi\left(F_{1}\right)-\Phi\left(F_{2}\right)=s_{U}^{F_{1}}-\sum_{U^{\prime} \in F_{2}} \alpha_{U^{\prime}} \cdot s_{U^{\prime}}^{F_{2}}
$$




$$
=\left\{s_{U}^{F_{1}}-t\right\}-\left\{\left(\sum_{U^{\prime} \in F_{2}} \alpha_{U^{\prime}} \cdot s_{U^{\prime}}^{F_{2}}\right)-t\right\},
$$

but the two terms within braces belong to $V / 2$. By symmetry and convexity, this difference is in $V$.

Claim 3.7. $\Phi$ is a convergent net.

Proof. We can assume that $\Phi(D)$ is infinite. By compactness of $Y$ we know that $\Phi(D)$ has an accumulation point $\tilde{k}$. We will show that $\tilde{k}$ is the limit of the net: let $W$ a symmetric convex neighborhood of 0 and let $F \in D$ be $W / 4$ fine. Since $\tilde{k}$ is an accumulation point, we may assume that $\Phi(F) \in \tilde{k}+W / 2$. By the previous claim, for every $F \preceq F^{\prime}$ we have that $\Phi(F)-\Phi\left(F^{\prime}\right) \in W / 2$, therefore $\Phi\left(F^{\prime}\right) \in \tilde{k}+W$. Therefore $\tilde{k}$ is a limit of the net. By Hausdorff, it is unique.

Claim 3.8. The limit of $\Phi$ does not depend on the choice of the points $s_{U}^{F}$.

Proof. let $\Psi$ be a function which is defined in the same way as $\Phi$ but choosing different points $r_{U}^{F}$ for the covers in $D$. Assume that $F$ is $W / 4$-fine for an arbitrary convex symmetric neighborhood of the origin $W$ and such that $\Phi(F) \in \tilde{k}+W / 2$, it follows then that $s_{U}^{F}-r_{U}^{F} \in W / 2$ which in turns yields to $\Phi(F)-\Psi(F) \in W / 2$, thus leading to $\Psi(F) \in \tilde{k}+W$, as desired.

Now, since the action provided by each element of $G$ over $X$ is continuous, we know that $\hat{g}: D \rightarrow D$ with $\hat{g}(F):=\left\{\pi_{g}(U) \mid U \in F\right\}$ defines a permutation of $D$ for every $g \in G$. The action of each element $g$ also permutes selection points for the open covers, this allows us to define the following function.

$$
\begin{aligned}
\Phi_{g}: D & \longrightarrow Y \\
F & \mapsto \sum_{U \in \hat{g}(F)} \mu_{U} \cdot\left(\pi_{g}\left(s_{g^{-1}(U)}^{F}\right)\right) .
\end{aligned}
$$

Claim 3.8 implies that $\Phi_{g} \rightarrow_{F} \tilde{k}$.

On the other hand we have

$$
\sum_{U \in \hat{g}(F)} \mu_{U} \cdot\left(\pi_{g}\left(s_{g^{-1}(U)}^{F}\right)\right)=\pi_{g}\left(\sum_{U \in \hat{g}(F)} \mu_{U} \cdot s_{g^{-1}(U)}^{F}\right)=\pi_{g}(\Phi(F)),
$$

showing that $g_{\tilde{k}} \Phi \rightarrow_{F} \tilde{h}$. Finally, the continuity of the action of $g$ implies that $g \Phi \rightarrow_{F} g \tilde{k}$ showing that $\tilde{k}$ is in fact a fixed point for the action.

$(2) \rightarrow(1)$ : By Theorem 2.17 we know that the space $C_{\sigma}(G)$ of bounded $\sigma$-continuous functions form a Banach vector space. Let $X:=C_{\sigma}(G)^{*}$ be

\footnotetext{
${ }^{2}$ Notice that if we fix the order on $F$ established in the definition of $\Phi$, when passing to $g F$ the associated values $\mu_{U}$ do not change since the given measure is $G$-invariant.
} 
its dual. By Alaoglu, the unit ball is a convex compact subset of $X$ with the weak* topology, and we can define a linear action of $G$ on $X$ by having, for any $g \in G$, any $F \in X$, and any $f \in C_{\sigma}(G)$,

$$
\pi_{g}(F)(f)=F\left({ }_{g} f\right)
$$

where

$$
{ }_{g} f(h)=f(g h)
$$

for any $h \in G$.

Claim 3.9. For a fixed $g$ we have $\pi_{g}: X \rightarrow X$ is continuous in the weak*topology.

Proof. Let $U$ be a sub-basic open set in $X$ with the weak* topology, so that $U:=\{F \mid F(f) \in B\}$ where $B$ is an open interval in $\mathbb{R}$. Then

$$
\left.\pi_{g}^{-1}(U)=\{F|g| f) \in B\right\}=\left\{F \mid F\left({ }_{g} f\right) \in B\right\}
$$

which is by definition another sub-basic open set in $X$.

Let $h \in G$ be any element. It is easy to see that the evaluation map $F_{h}(f)=f(h)$ is in $X$.

Claim 3.10. For every $h \in G$, the map $F_{h}$ is $\sigma$-continuous.

Proof. Once again, let $U$ be a sub-basic open set in $X$ of the form

$$
U:=\{F \mid F(f) \in B\}
$$

for $B$ an open interval in $\mathbb{R}$. By definition,

$$
F_{h}^{-1}(U):=\left\{g \in G \mid F_{h}(g) \in B\right\}=\{g \in G \mid f(g h) \in B\} .
$$

Since $f$ is $\sigma$-continuous we can find a countable family $\left\{D_{i}\right\}_{i<\omega}$ of definable subsets

$$
\bigcup_{i \in \omega} D_{i}=f^{-1}(U)
$$

and

$$
F_{h}^{-1}(U)=\left(f^{-1}(U)\right) h^{-1}=\left(\bigcup_{i \in \omega} D_{i}\right) h^{-1}=\bigcup_{i \in \omega} D_{i} h^{-1}
$$

which is a countable union of definable sets, as required.

It follows that the action of $G$ on the unit ball of $X$ has a fixed point $F$. The characteristic functions of definable sets are $\sigma$-continuous, so we know that we can define a finitely additive measure on definable subsets of $G$ by $\mu(D)=F\left(\mathbf{1}_{D}\right)$. But then

$$
\mu(g D)=F\left(\mathbf{1}_{g D}\right)=F\left({ }_{g} \mathbf{1}_{D}\right)={ }_{g} F\left(\mathbf{1}_{D}\right)=F\left(\mathbf{1}_{D}\right)
$$

by invariance, thus proving definable amenability of $G$. 
10JUAN FELIPE CARMONA, KEVIN DÁVILA, ALF ONSHUUS, AND RAFAEL ZAMORA

To prove $(1) \rightarrow(2)$ we only used that $\pi_{k}$ was measurable. Since any $\sigma$ continuous function is a measurable function, we have the following equivalent statement.

Theorem. The following are equivalent:

(1) $G$ is an $\omega_{1}$-saturated definably amenable group.

(2) If $\pi$ is a linear action of $G$ into a convex compact subset $Y$ of a locally convex topological vector space $V$ such that

(a) $\pi_{g}$ is continuous for every $g \in G$.

(b) For some $k \in Y$ the function $\pi_{k}$ is a measurable function from the $\sigma$-algebra of definable sets in $G$ into the Borel $\sigma$-algebra of $Y$.

Then $\pi$ has a fixed point.

\section{REFERENCES}

[1] Chernikov, A., Simon, P. (2015). Definably amenable NIP groups. arXiv preprint arXiv:1502.04365.

[2] Day, Mahlon M. Fixed-point theorems for compact convex sets. Illinois Journal of Mathematics 5.4, 1961. pp. 585-590.

[3] Gismatullin, Jakub ; Penazzi, Davide ; Pillay, Anand. Some model theory of SL(2, $\mathbb{R})$. Fund. Math. 229 (2015), no. 2, 117-128.

[4] Hrushovski, Ehud ; Peterzil, Ya'acov ; Pillay, Anand . Groups, measures, and the NIP. J. Amer. Math. Soc. 21 (2008), no. 2, 563-596.

[5] Poizat, Bruno . Groupes stables. Une tentative de conciliation entre la geometrie algebrique et la logique mathematique. [An attempt at reconciling algebraic geometry and mathematical logic] Nur al-Mantiq wal-Marifah [Light of Logic and Knowledge], 2. Bruno Poizat, Lyon, 1987. vi+218 pp. ISBN: 2-9500919-1-1

[6] Wagon, Stan. The Banach-Tarski paradox. With a foreword by Jan Mycielski. Corrected reprint of the 1985 original. Cambridge University Press, Cambridge, 1993. xviii+253 pp. ISBN: 0-521-45704-1

Universidad Antonio Nariño, Calle 58 A No. 37 - 94, Bogotá, Colombia

E-mail address: jfcarmonag@gmail.com

Fundación Universidad del Norte, Km.5 Vía Puerto Colombia, Barranquilla, Colombia

E-mail address: kevindavilacastellar@gmail.com

Universidad de los Andes, Cra 1 No 18A-10, Bogotá, Colombia

E-mail address: aonshuus@uniandes.edu.co

Escuela de Matemática, Universidad de Costa Rica, San Pedro, San Jose, Costa RicA

E-mail address: rafael.zamora_c@ucr.ac.cr 